\title{
Prophylaxis of Venous Thromboembolism in Patients with Infectious Disease
}

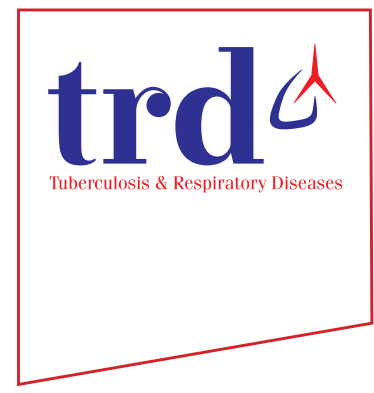

\author{
Hun-Gyu Hwang, M.D., Ph.D. ${ }^{1}{ }^{(i)}$, Yang-Ki Kim, M.D., Ph.D. ${ }^{2}$, Myung-Shin Kim, M.D., Ph.D. ${ }^{1}$, Gune- \\ Il Lim, M.D., Ph.D. ${ }^{1}$ and Sam Schulman, M.D., Ph.D. ${ }^{3,4}$ \\ ${ }^{1}$ Division of Respiratory and Allergy Medicine, Department of Internal Medicine, Soonchunhyang University Gumi Hospital, \\ Gumi, ${ }^{2}$ Division of Respiratory and Allergy Medicine, Department of Internal Medicine, Soonchunhyang University Seoul \\ Hospital, Seoul, Korea, ${ }^{3}$ Division of Hematology and Thromboembolism, Department of Medicine, Thrombosis and \\ Atherosclerosis Research Institute, Hamilton Health Sciences (Hamilton General Site), McMaster University, Hamilton, ON, \\ Canada, ${ }^{4}$ Department of Obstetrics and Gynecology, I.M. Sechenov First Moscow State Medical University, Moscow, Russia
}

Venous thromboembolism (VTE), defined as deep vein thrombosis and/or pulmonary embolism (PE), is associated with a significant disease entity in medically ill patients ${ }^{1}$. The incidence of VTE is increasing gradually in Asia even though it is still lower than in Western countries ${ }^{2-4}$. The incidence of VTE increases sharply in the elderly ${ }^{5}$ of which the proportion is expected to rise more rapidly in South Korea than hitherto ${ }^{6}$. Moreover, recent studies showed that the recurrence rate of VTE in Korea is comparable to the rate in Western societies ${ }^{3,7}$. Thus, VTE might become a big burden for national health care in near future in South Korea.

What kind of efforts are needed to minimize future risk from VTE at this moment? It is timely to focus on identifying patients at high risk of VTE in advance and applying prophylaxis, beyond treating patients diagnosed with VTE, because both physicians' and patients' awareness increased since introduction of direct oral anticoagulant in 2013. The next step would be to find out the most appropriate candidates for prevention of VTE among medically ill patients.

Who are those candidates? Many studies showed that

Address for correspondence: Hun-Gyu Hwang, M.D., Ph.D.

Division of Respiratory and Allergy Medicine, Department of Internal

Medicine, Soonchunhyang University Gumi Hospital, 1791 Gongdan-ro, Gumi 39371, Korea

Phone: 82-54-468-9040, Fax: 82-54-468-9051

E-mail: hwangpark@hanmail.net

Received: Mar. 22, 2020

Revised: Mar. 27, 2020

Accepted: Mar. 30, 2020

Published online: Apr. 29, 2020

(9) It is identical to the Creative Commons Attribution Non-Commercial License (http://creativecommons.org/licenses/by-nc/4.0/). about $40 \%$ of the medical hospitalized patients face a moderate or high risk to develop $\mathrm{VTE}^{1}$ during which acute infectious disease, age older than 75 years, cancer, and previous VTE history are well-known risk factors ${ }^{8}$. Clayton et al. ${ }^{9}$ showed that especially, preceding respiratory infection may increase the risk of PE or VTE in the 3 months following infection (adjusted odds ratio, 2.50; 95\% confidence interval, 1.33-4.72) from a primary care general practice database. Recently, Lee et al. ${ }^{10}$ reported that infection combined with VTE increased mortality. However, there was the limitation that PE was diagnosed incidentally by conventional computed tomography in over two-thirds of the patients. That is why they could not prove the temporal and causal relationship between infection and PE.

Let us look at recent studies ${ }^{11,12}$, which assessed whether thromboprophylaxis decrease the rate of VTE in medically ill patients including acute infection or respiratory disease, while the implementation of prevention programs decreased the rate of VTE in surgical ${ }^{13}$ and orthopedic department ${ }^{14}$. Cohen et al. ${ }^{11}$ showed in the MAGELLAN trial that the patients with infection, respiratory insufficiency and two or more medical condition constituted $45.8 \%, 27.3 \%$, and $30.6 \%$, respectively, in rivaroxaban group in which extended duration of prophylaxis reduced the risk of VTE, but rivaroxaban was also associated with an increased risk of bleeding. Spyropoulos et al. ${ }^{12}$ included in the MARINER trial patients with infectious disease $(17.5 \%)$ and respiratory insufficiency $(26.2 \%)$ in the rivaroxaban group versus placebo given to medical patients for 45 days after hospital discharge, in which rivaroxaban was not associated with a significant lower risk of symptomatic VTE and death due to VTE versus placebo. Spyropoulos et al. ${ }^{12}$ estimated the risk for VTE on the basis of a modified International Medical Prevention Registry on Venous Thromboembolism (IMPROVE) score of 4 or higher or a risk score of 2 or 3 plus a plasma D-dimer level of more than twice the upper limit of the normal range. Over one-third patients had IMPROVE score of 4 or higher. 
In real-life observational study, Mahlab-Guri et al. ${ }^{15}$ reported that thromboprophylaxis did not have significant effect on reducing the number of VTE events, even though the rate of patients treated for thromboprophylaxis increased from $22 \%$ at 2013 to $46 \%$ at 2018. The risk of VTE was calculated with Padua score which includes malignancy, previous VTE, obesity, elderly age, bedrest, acute infection and so on ${ }^{15}$. About $30.1 \%$ had a Padua score of 4 points or more (representing high risk of VTE), which is indication for VTE prophylaxis. In this study, $25.1 \%$ of patients had infectious disease, but there was no analysis to assess the outcome in the subgroup of patients with infectious disease ${ }^{15}$.

In clinical practice in South Korea, the proportion of patients receiving prophylaxis may be much lower than $46 \%$ although there is lack of exact data. We need to increase the rate of prophylaxis in genuine candidates with high risk of VTE (Padua score of 4 points or more). There are still controversies about the most appropriate candidates and duration for prophylaxis of VTE in medically ill patients in recent studies ${ }^{11,12,15}$. We have to make our own efforts to identify candidates for VTE prophylaxis in retrospective and prospective studies in South Korea. These efforts may increase the rate of prophylaxis in adequate candidate, and after all reduce the rate of VTE in medical patients admitted with comorbidity in a super-aged Korean society before long.

\section{Conflicts of Interest}

No potential conflict of interest relevant to this article was reported.

\section{References}

1. Dobromirski M, Cohen AT. How I manage venous thromboembolism risk in hospitalized medical patients. Blood 2012;120:1562-9.

2. Jang MJ, Bang SM, Oh D. Incidence of venous thromboembolism in Korea: from the Health Insurance Review and Assessment Service database. J Thromb Haemost 2011;9:85-91.

3. Hwang HG, Choi WI, Lee B, Lee CW. Incidence and risk factors of recurrent venous thromboembolism after pulmonary embolism. Tuberc Respir Dis 2019;82:341-7.

4. Hwang HG, Schulman S. Respiratory review of 2013: pulmonary thromboembolism. Tuberc Respir Dis 2013;75:89-94.

5. Engbers MJ, van Hylckama Vlieg A, Rosendaal FR. Venous thrombosis in the elderly: incidence, risk factors and risk groups. J Thromb Haemost 2010;8:2105-12.

6. Kim H, Jeon B. Developing a framework for performance assessment of the public long-term care system in Korea: methodological and policy lessons. Health Res Policy Syst 2020;18:27.

7. Kim YK. Predictors of recurrent venous thromboembolism after pulmonary embolism in Korea. Tuberc Respir Dis 2019;82:357-8.

8. Alikhan R, Cohen AT, Combe S, Samama MM, Desjardins L, Eldor A, et al. Risk factors for venous thromboembolism in hospitalized patients with acute medical illness: analysis of the MEDENOX Study. Arch Intern Med 2004;164:963-8.

9. Clayton TC, Gaskin M, Meade TW. Recent respiratory infection and risk of venous thromboembolism: case-control study through a general practice database. Int J Epidemiol 2011;40:819-27.

10. Lee GD, Ju S, Kim JY, Kim TH, Yoo JW, Lee SJ, et al. Risk factor and mortality in patients with pulmonary embolism combined with infectious disease. Tuberc Respir Dis 2020;83: 157-66.

11. Cohen AT, Spiro TE, Buller HR, Haskell L, Hu D, Hull R, et al. Rivaroxaban for thromboprophylaxis in acutely ill medical patients. N Engl J Med 2013;368:513-23.

12. Spyropoulos AC, Ageno W, Albers GW, Elliott CG, Halperin JL, Hiatt WR, et al. Rivaroxaban for thromboprophylaxis after hospitalization for medical illness. N Engl J Med 2018;379:1118-27.

13. Alikhan R, Peters F, Wilmott R, Cohen AT. Fatal pulmonary embolism in hospitalised patients: a necropsy review. J Clin Pathol 2004;57:1254-7.

14. Cohen AT, Edmondson RA, Phillips MJ, Ward VP, Kakkar VV. The changing pattern of venous thromboembolic disease. Haemostasis 1996;26:65-71.

15. Mahlab-Guri K, Otman MS, Replianski N, Rosenberg-Bezalel S, Rabinovich I, Sthoeger Z. Venous thromboembolism prophylaxis in patients hospitalized in medical wards: a real life experience. Medicine (Baltimore) 2020;99:e19127. 